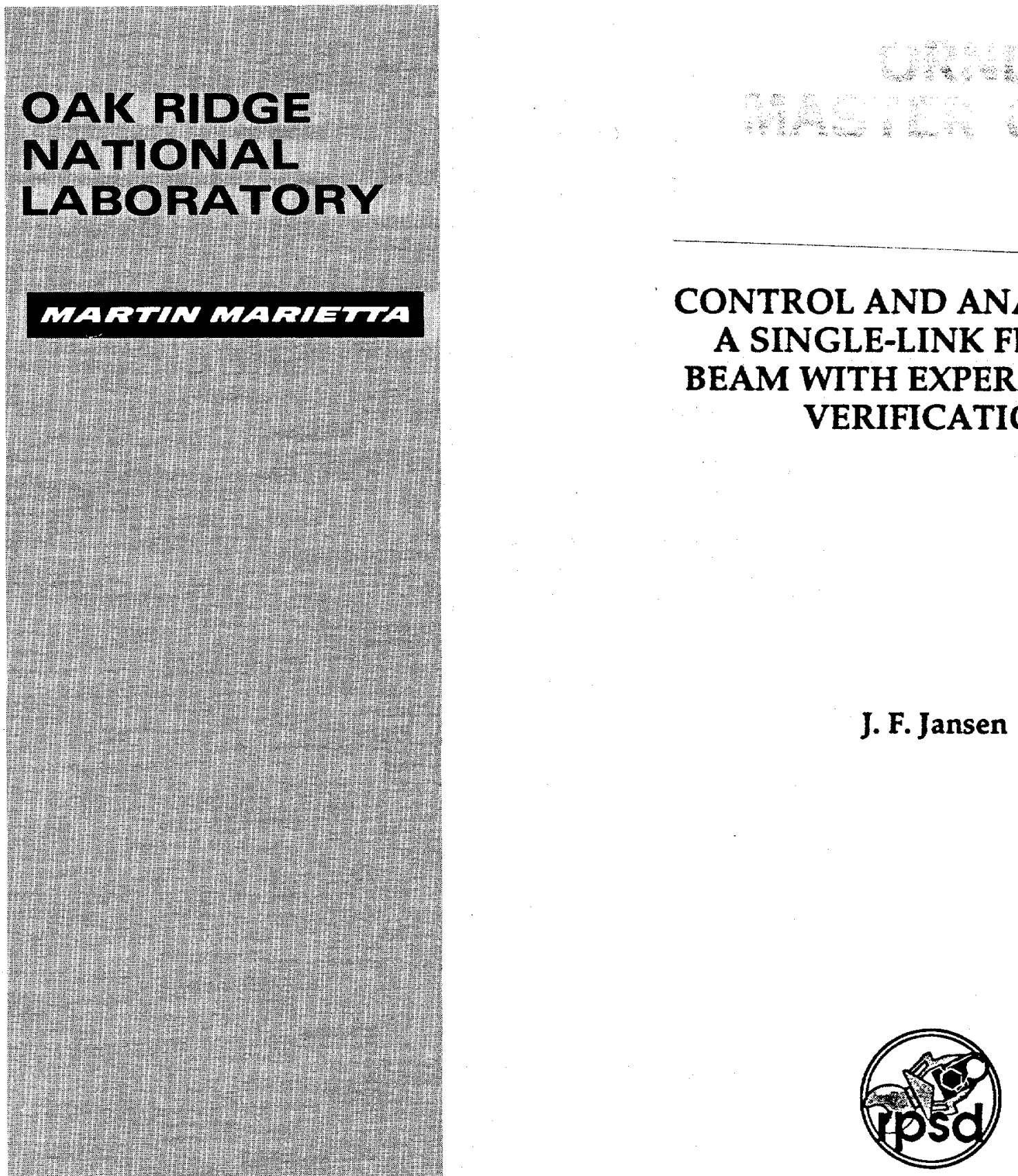


This report has been reproduced directly from the best available copy.

Available to DOE and DOE contractors from the Office of Scientific and Technical Information, P.O. Box 62, Oak Ridge, TN 37831; prices available from (615) 576-8401, FTS 626-8401.

Available to the public from the National Technical Information Service, U.S. Department of Commerce, 5285 Port Royal Rd., Springfield, VA 22161.

This report was prepared as an account of work sponsored by an agency of the United States Government. Neither the United States Government nor any agency thereof, nor any of their employees, makes any warranty, express or implied, or assumes any legal liability or responsibility for the accuracy, completeness, or usefulness of any information, apparatus, product, or process disclosed, or represents that its use would not infringe privately owned rights. Reference herein to any specific commercial product, process, or service by trade name, trademark, manufacturer, or otherwise, does not necessarily constitute or imply its endorsement, recommendation, or favoring by the United States Government or any agency thereof. The views and opinions of authors expressed herein do not necessarily state or reflect those of the United States Government or any agency thereof. 
Office of Technology Department

Robotics Technology Development Program

\title{
CONTROL AND ANALYSIS OF A SINGLE-LINK FLEXIBLE BEAM WITH EXPERIMENTAL VERIFICATION
}

\author{
J. F. Jansen \\ Robotics \& Process Systems Division
}

Date Published-December 1992

Prepared by the OAK RIDGE NATIONAL LABORATORY

Oak Ridge, Tennessee 37831 managed by

MARTIN MARIETTA ENERGY SYSTEMS, INC. for the U.S. DEPARTMENT OF ENERGY under contract DE-AC05-84OR21400 


\section{CONTENTS}

Section

Page

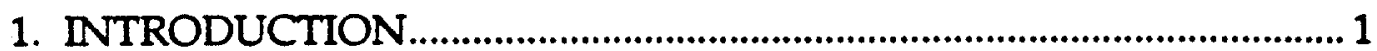

2. GENERAL PROBLEM STATEMENT …................................................... 2

3. BENCH-SCALE MODEL DESCRIPTION …........................................... 5

4. ROBUST PRESHAPING............................................................................. 7

5. ADAPTIVE INTERFERENCE CANCELER ............................................... 11

6. ACCELERATION FEEDBACK ................................................................. 15

7. CONCLUSION AND SUMMARY …….................................................... 19

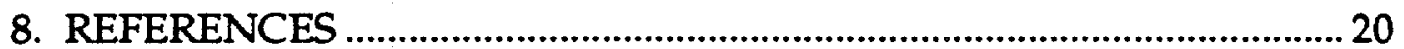




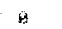




\section{Control and Analysis of a Single-Link Flexible Beam with Experimental Verification}

\section{INTRODUCTION}

The objective of this report is to ascertain the general conditions for the avoidance and reduction of residual vibration in a flexible manipulator. Conventional manipulators usually have a 1.5 to $2-\mathrm{m}$ reach, and their associated dynamic models typically are composed of lumped parameter elements; the major compliance emanates from the drive trains because of torsional loading effects. The energy storage of the drive system is predominantly potential energy because of the low inertia in the drive train; thus simple spring models have been adequate.

A long-reach manipulator with a large aspect ratio (length to diameter) is a fundamentally different problem. Energy storage for this type of manipulator is distributive by nature because of the potential energy resulting from bending and the kinetic energy due to deflection rates. Instead of ordinary differential equations, partial differential equations are required to describe this system, making the analysis more difficult. The general flexibility problem associated with a distributive dynamic system, with specific emphasis on flexible manipulator, will be addressed in this report. Furthermore, three control schemes will be discussed and demonstrated on a single flexible manipulator to determine their general merits. 


\section{GENERAL PROBLEM STATEMENT}

In this section the general conditions pertaining to the avoidance and reduction of residual vibrations in a linear distributive dynamic system will be discussed. From Meirovitch (1967), the general formulation for the continuous undamped distributive parameter system can be described as

$$
L[w(P, t)]+M(P) \frac{\partial^{2} w(P, t)}{\partial t^{2}}=f(P, t)+F_{j}(t) \delta\left(P-P_{j}\right)
$$

over some domain D. In Eq. (1), L is the linear, homogenous, self-adjoint, positive-definite, spatial, differential operator with derivatives up to order $2 \mathrm{p}$; $\mathrm{P}$ represents the spatial coordinates; $\mathrm{M}$ is a function of $\mathrm{P}$ and contains information on the mass distribution of the system; $f$ represents the distributed forces; $F_{j}$ represents one of $l_{f}$ distinct concentrated forces at points $P_{j} ; \delta$ is the Dirac's delta function; and $w(P, t)$ is the displacement at $P$ at time $t$. At every point of the boundary, there are $\mathrm{p}$ boundary conditions of the type

$$
B_{i}[w(P, t)]=0, \quad i=1,2, \ldots, p,
$$

where $B_{i}$ represents linear, homogenous, differential operators containing derivatives of order up through $2 p-1$. Further, typically, shear and rotatory inertia terms are ignored in Eq. (1), and linearization of the dynamic system has occurred, which means that Eq. (1) is an approximation at best.

By means of modal analysis (Meirovitch 1967), Eq. (1) can be transformed into the following infinite set of uncoupled ordinary differential equations:

$$
\ddot{x}_{i}+w_{i}^{2} x_{i}=f_{i}(t) \quad i=1,2,3, \ldots,
$$

where

$\mathbf{x}_{\mathbf{i}}=$ ith generalized coordinate;

$$
w(P, t)=\sum_{i=1}^{\infty} w_{i}(P) x_{i}(t)
$$

$w_{i}=i$ th eigenfunction (or mode shape) associated with the problem

$L\left[w_{i}\right]=\omega_{i}^{2} M w_{i}$, which also satisfies all of the boundary conditions

$\mathrm{B}_{\mathrm{j}}\left[\mathbf{w}_{\mathrm{i}}\right]=0$; 


$$
f_{i}(t)=\int_{0} w_{i}(P) f(P, t) d D(P)+\sum_{j=1}^{l_{t}} w_{i}(P) F_{j}(t) .
$$

Equation (3a) can be converted into state variable form with the usual approximation of truncating the series to include $n$ modes and by including damping terms $\zeta_{i}$ as follows:

$$
\frac{d}{d t}\left[\begin{array}{c}
x_{0} \\
\dot{x}_{0} \\
x_{1} \\
\dot{x}_{1} \\
\vdots \\
\dot{x}_{n}
\end{array}\right]=\left[\begin{array}{cccccc}
0 & 1 & & & & \\
-\omega_{0}^{2} & -2 \zeta_{0} \omega_{0} & & & & \\
& & 0 & 1 & & \\
& & -\omega_{1}^{2} & -2 \zeta_{1} \omega_{1} & & \\
& & & & \ddots & \\
& & & & -\omega_{n}^{2} & -2 \zeta_{n} \omega_{n}
\end{array}\right]\left[\begin{array}{c}
x_{0} \\
\dot{x}_{0} \\
x_{1} \\
\dot{x}_{1} \\
\vdots \\
\dot{x}_{n}
\end{array}\right]+\left[\begin{array}{c}
0 \\
f_{1}(t) \\
0 \\
f_{2}(t) \\
\vdots \\
f_{n}(t)
\end{array}\right]
$$

or in more compact matrix notation:

$$
\dot{\bar{x}}=A \bar{x}+\bar{f} \text {. }
$$

For the types of problems addressed in this paper, the eigenvalues $p_{i}$ (for $i=1, \ldots, 2 n$ ) associated with Eq. (4b) are usually distinct, which means that a complete set of eigenvectors, $\bar{v}_{\mathbf{i}}$, can be found such that Eq. (4b) can be transformed into the standard uncoupled canonical form

$$
\overline{\mathbf{z}}=\mathrm{D} \overline{\mathbf{z}}+\overline{\mathbf{u}},
$$

where

$$
\begin{aligned}
& \bar{x}=T \bar{z}, \\
& D=\operatorname{diag}\left(p_{i}\right), \\
& \bar{u}=T^{-1} \bar{f}, \\
& T=\left[\bar{v}_{i}\right] .
\end{aligned}
$$

The solution to Eq. (5) at time $\mathrm{T}$ is simply

$$
z_{i}(T)=e^{p T} z_{i}(0)+e^{P T} \int_{0}^{T} e^{-p i \tau} u_{i}(\tau) d \tau \text { for } i=1,2, \ldots, 2 n .
$$


If we let the input $u_{i}$ be a time-bounded function (i.e., $u_{i}(t)=0$ ) for time outside of $0<\mathrm{t}<\mathrm{T}$, and assuming that all initial conditions are zero, Eq. (6) can be modified to

$$
\int_{0}^{-} e^{-p_{i} \tau} u_{i}(\tau) d \tau=e^{-p_{i} T_{Z_{j}}(T)}
$$

The left side of Eq. (7a) is the Laplace transform of $u_{i}$ evaluated at $p_{i} ;$ i.e.,

$$
\left.U_{i}(s)\right|_{s=p_{i}}=\int_{0}^{-} e^{-p^{\tau} \tau} u_{i}(\tau) d \tau=e^{-p_{i} T_{Z_{k}}(T)} .
$$

For the final state $z_{i}(T)$ to be equal to zero (i.e., no residual vibration) then $\left.\mathrm{U}_{\mathrm{i}}(\mathrm{s})\right|_{\mathrm{s}=\mathrm{p}}$ must be zero. This means that the Laplace transform of the inputs $\left(\mathrm{f}_{\mathrm{i}} \mathrm{s}\right)$ in Eq. (3c) must be zero when evaluated at the poles of the dynamic system. This is exactly the condition stated by Bhat and Miu (1990), where they proved, even for the case for repeated roots, "that the necessary and sufficient condition for zero residual vibration is that the Laplace transform of the time bounded control input have zero component at the system poles." Furthermore, they mention that, "if the system has non-zero damping, then zero contribution at the system resonant frequency does not guarantee zero residual vibration." This last point is particularly important because in practice the resonant frequencies of a dynamic system are typically known to within a certain precision bound; however, the damping is typically not known, nonlinear by nature, or too troublesome to obtain.

The preceding discussion addresses the problem of how to avoid creating residual vibrations in a flexible system and is basically an open-loop method. The second problem to be addressed in this report is how one can add system damping if residual vibrations already exist in the system. Two possible approaches exist. The first approach can be found in Jayasuriya and Choura (1990) where an input is generated based on perfect knowledge of the model and of the initial conditions of the system. After a finite time duration, all of the residual vibrations will go to zero. This scheme is again basically an open-loop scheme. The second approach is by means of closed-loop feedback using some type of measuring device (e.g., accelerometer, strain gauge, optical) on the beam. Many schemes are proposed to address this problem and are documented (Jansen et al. 1992). The intent of this report is to examine two closed-loop schemes that can easily be implemented on a multi-link flexible manipulator. A couple of unique solutions to the problem will be addressed and are detailed in a later section of this report. 


\section{BENCH-SCALE MODEL DESCRIPTION}

A single-link flexible manipulator has been constructed (see Fig. 1) to examine various control schemes in order to ascertain their potential for long-reach manipulators. The servo for the beam is a brush type dc motor with a 65:1 gear ratio. Approximately 10 to $20 \%$ of the motor torque is required to overcome the friction (coulomb, stiction, and linear friction) at the gear and motor. The block at the end of the beam is an accelerometer that will be used to feed data back from the tip of the beam. A joint controller based on the classical proportionalderivative (PD) controller is implemented to drive the beam. Position and velocity at the joint are sensed by a resolver. Torque is sensed by means of a strain gauge located near the hub of the beam (not shown in Fig. 1). This torque signal is used by an inner joint torque control loop whose main purpose is to increase the backdrivability (i.e., to reduce friction effects and to improve linearity of joint dynamics) of the drive train and to reduce motor torque ripple effects.

The beam transfer functions relating hub torque to tip position and joint position have been derived for the uniform beam case (Jansen et al. 1992). For a mass at the end of the beam, similar canonical forms are found and can be expressed as

$$
H_{u}^{y}=\frac{N_{u}^{y}}{D_{u}^{y}}=\frac{K_{d c} \prod_{i=1}^{n}\left[\frac{s^{2}}{\omega_{z i}^{2}}+\frac{2 \delta_{z i} s}{\omega_{z i}}+1\right]}{s_{j=1}^{2}\left[\frac{s^{2}}{\omega_{p j}^{2}}+\frac{2 \delta_{p j} s}{\omega_{p j}}+1\right]}
$$

for tip position and

$$
H_{u}^{\theta}=\frac{N_{u}^{\theta}}{D_{v}^{\theta}}=\frac{K_{d c}^{\prime} \prod_{i=1}^{n}\left[\frac{s^{2}}{\omega_{z i}^{\prime 2}}+\frac{2 \delta_{z i}^{\prime} s}{\omega_{z i}^{\prime}}+1\right]}{s_{j=1}^{2}\left[\frac{s^{2}}{\omega_{p j}^{2}}+\frac{2 \delta_{p j} s}{\omega_{p j}}+1\right]}
$$

for hub position. 


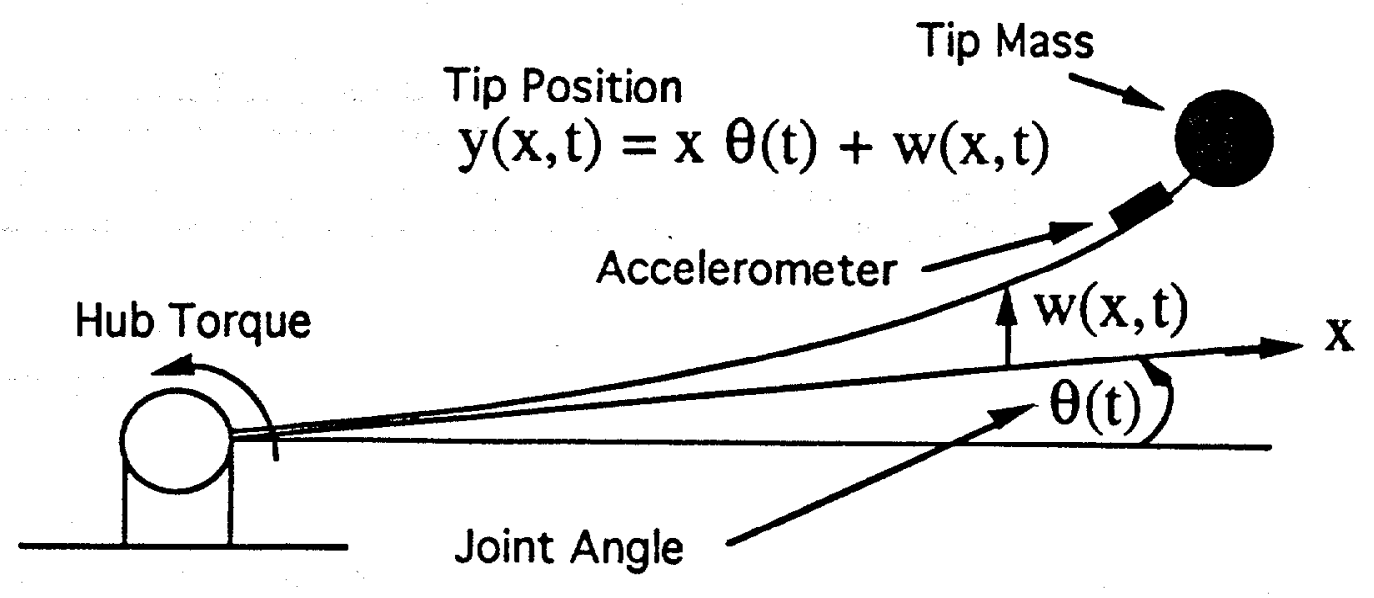

Fig. 1. Flexible beam model. 


\section{ROBUST PRESHAPING}

As previously mentioned, the poles of the dynamic system are typically not precisely known. Usually, the resonant frequencies are known to within some tolerance. To avoid residual vibrations, the Laplace transform of the path trajectory must equal zero when evaluated at the poles of the dynamic system. When the poles lie on the $j \omega$ axis, the approach is identical to filtering out the $j \omega$ frequency components of the path trajectory $p(t)$. If the poles do not lie on the jw axis and the exact location of the poles of the dynamic system is unknown, then the standard notch filter will leave some residual vibrations. For this report, the assumption is made that only the resonant frequencies of the system are known; the damping ratios are unknown and assumed to be zero. To add robustness to the notch filter, the shape of $\mid P(s) \backslash$ where $P(s)=\langle\{p(t)\}$, needs to flatten around the resonant frequencies of the system so as to diminish the residual vibrations (Bhat and Miu 1990). To achieve this flattening, the first $n$ derivatives of $P(s)$ should be equal to zero at the resonant frequencies. As the value of $n$ becomes larger, the less sensitive the system becomes to the lack of precise knowledge of the beam damping and resonant frequencies. This approach, which is formulated in the $s$ domain, has similarities with the time domain approach proposed by Singhose et al. (1990), and the interested reader can refer to Murphy and Watanabe (1992) for details. From a robustness perspective, it is sufficient to set the first derivative to zero for the one-link flexible manipulator. This is accomplished by setting the zero of the notch filter to have multiplicity of 2 . The proposed filter presented is called a robust notch filter (RNF) to highlight its purpose of diminishing the effect of not knowing the exact pole location of the dynamic system. The RNF for each resonant frequency is selected as

$$
F(s)=\frac{\left[\left(\frac{s}{\omega_{2}}\right)^{2}+1\right]^{2}}{\left[\left(\frac{s}{\omega_{p}}\right)^{2}+2 \frac{\zeta_{p}}{\omega_{p}} s+1\right]^{3}},
$$

where

$$
\begin{aligned}
\omega_{z}= & \text { zero resonant frequency, } \\
\omega_{p}= & \text { low-pass filter natural frequency, and } \\
\zeta_{p}= & \text { damping ratio (set to } 1 \text { to achieve an overdamped } \\
& \text { response). }
\end{aligned}
$$

The order of the denominator of Eq. (8) was intentionally set higher than the numerator to cause the filter to have a $12-\mathrm{d}$ B-per-octave roll-off at higher 
frequencies. Although the $\omega_{z}$ term in Eq. (8) is set to the resonant natural frequency of the system, the low-pass filter natural frequency $\omega_{\mathrm{p}}$ cannot be selected independently of $\omega_{2}$. If $\omega_{p}$ is chosen much larger than $\omega_{2}$ then the numerator term dominates in the frequency range below $\omega_{\mathrm{p}}$ making the numerator essentially a fourth-order differentiator at frequencies from $\omega_{z}$ to $\omega_{\mathrm{p}}$. Any discontinuities in the path, including up to its fourth derivative, could produce large-magnitude oscillations in the system. To avoid this problem, $\omega_{\mathrm{p}}$ was set equal to $\omega_{\mathrm{z}}$.

To determine the resonant frequencies of the beam, an accelerometer sensor and a fast Fourier transform (FFT) software routine were used to generate the power spectrum density curve shown in Fig. 2. (The units for the ordinate axis have been modified by an arbitrary ratio so that the peaks are clear.) The beam was slewed $20^{\circ}$ over $0.25 \mathrm{~s}$ to excite the beam vibrational modes. These resonant frequencies should correspond to the pin-free boundary conditions. The significant resonant frequencies of the beam are determined from this curve.

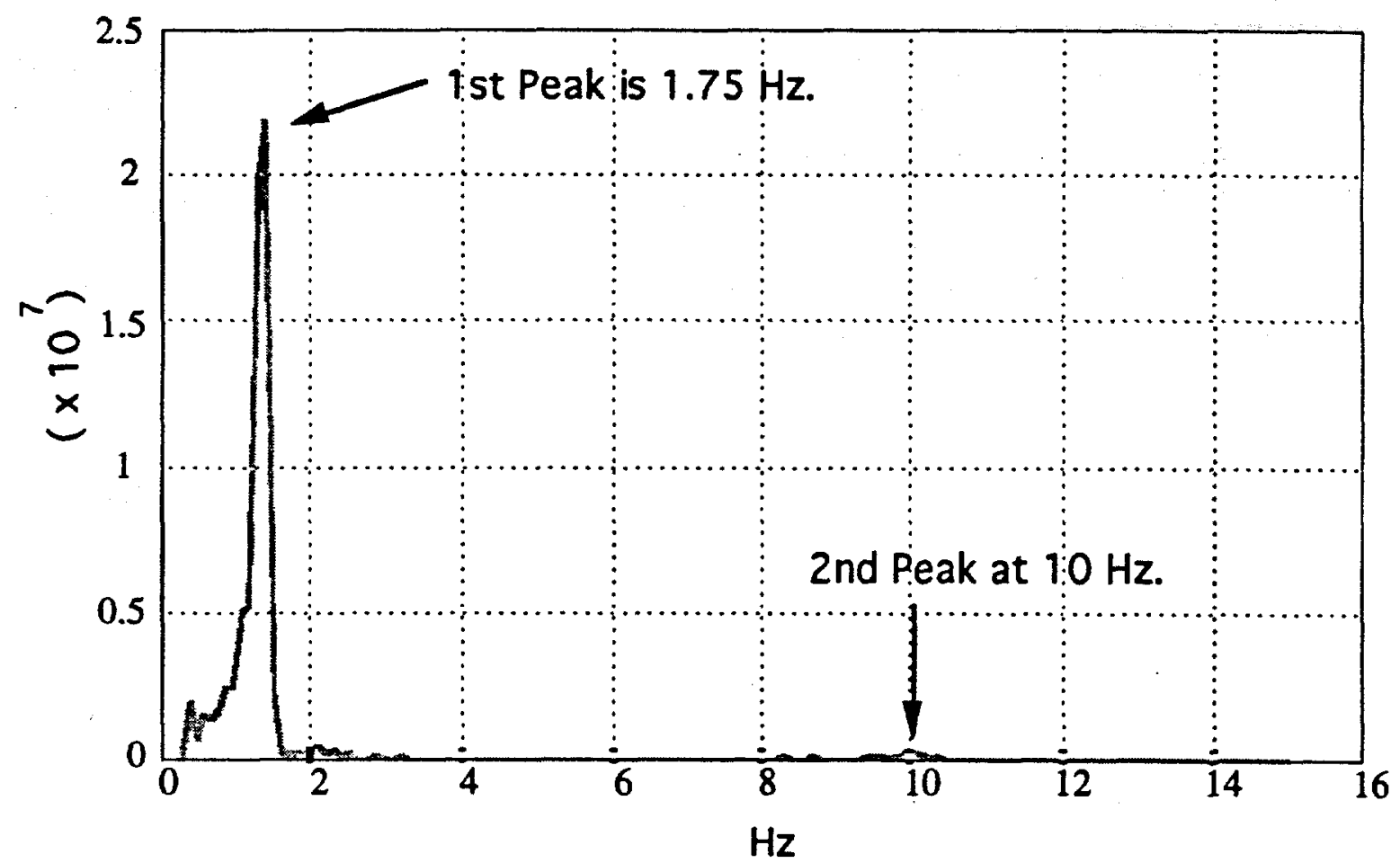

Fig. 2. Power spectrum density of accelerometer sensor (the numerical values for the vertical scale are relative). 
The first resonant peak is approximately $1.75 \mathrm{~Hz}$; the second peak is approximately $10 \mathrm{~Hz}$. Since the first resonant peak is dominant, only the first resonant frequency was "notched" out of the planned path. To notch out other frequency components, one merely has to cascade multiple RNFs together.

Figure 3 shows the basic block diagram to implement the shaped-input method based on the RNF method where the $\mathrm{ZOH}$ block represents a zero order hold and the PWM block represents a pulse-width modulator.

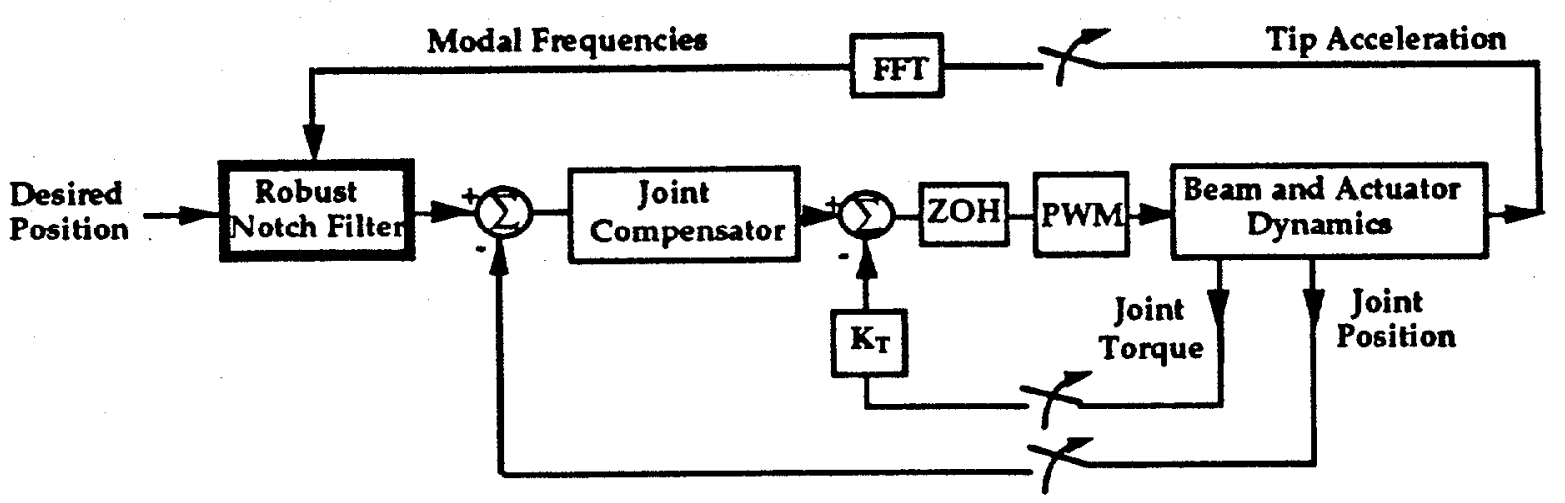

Fig. 3. Shaped-input block diagram.

The FFT block in Fig. 3 is updated at a much lower rate than the other feedback loops because its function is to find the resonant frequencies of the beam. Update rates of $5 \mathrm{~s}$ are feasible with standard digital signal-processing boards. The modal frequency locations are not known with any great precision, allowing the FFT to update the modal frequencies at a 5-s rate without difficulty. For a single-link beam, the modal frequency locations only change because of loading; however, for a multi-link beam, both the workspace geometry and loading influence their locations.

Acceleration data were used to compare the performance of a single flexible beam with and without preshaping of the planned trajectory. The flexible manipulator was slewed $20^{\circ}$ for a duration of $0.25 \mathrm{~s}$, as shown in Fig. 4 . Acceleration data were integrated to obtain relative tip velocity. On the graphs showing tip velocity, $1 \mathrm{~g}$ (gravitational acceleration of $9.8 \mathrm{~m} / \mathrm{s}^{2}$ ) corresponds to 256.8 binary counts (cts); counts seconds (cts*s) correspond to $0.038 \mathrm{~m} / \mathrm{s}$ ). A large tip vibration is apparent after the slewing motion has been completed. $A$ small residual motion is always present because of gravitational load variations (the beam is slewed perpendicular to the gravitation potential field) and because of the nonlinear friction in the drive train (reduced by the inner torque loop). 


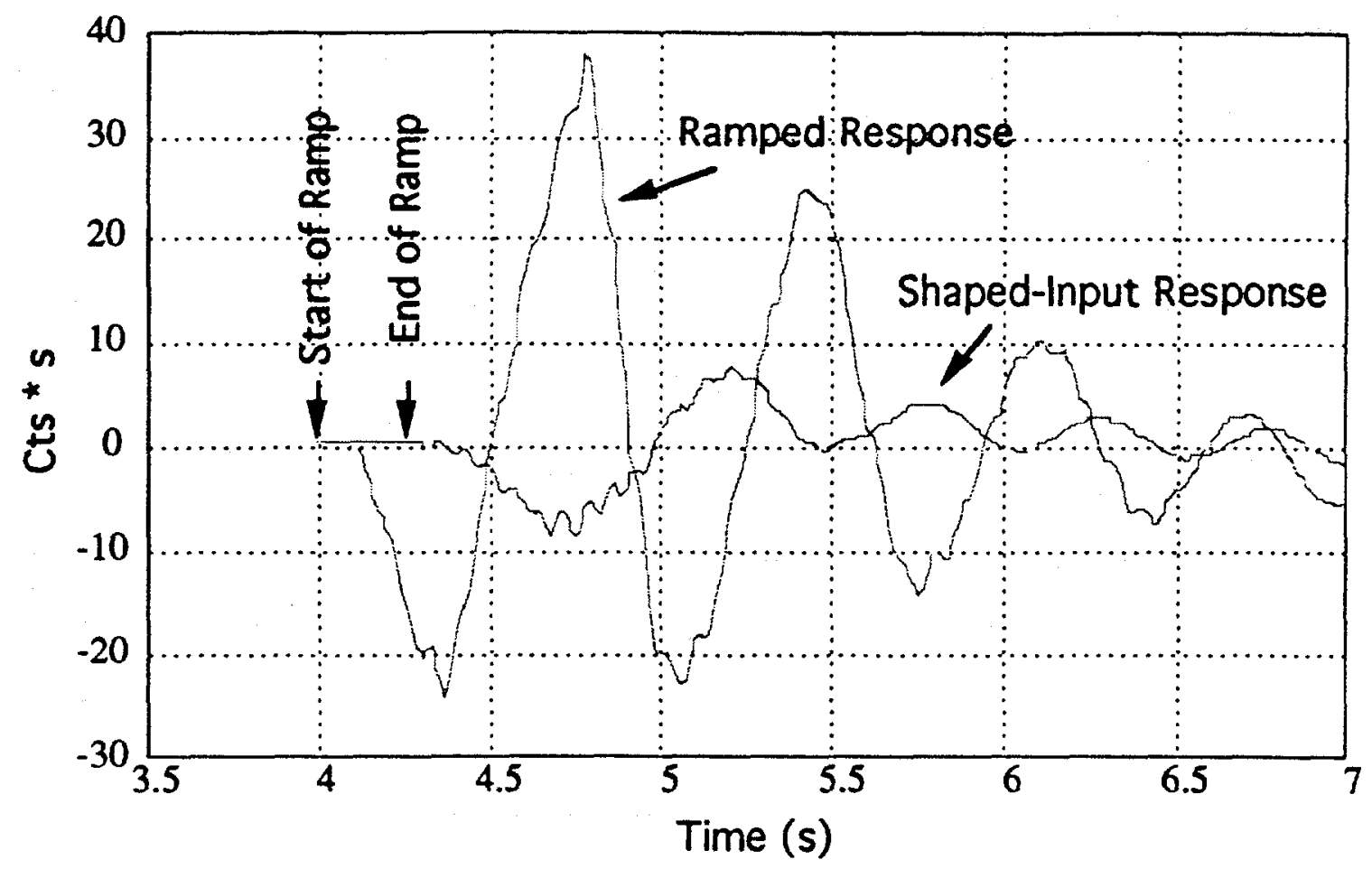

Fig. 4. Shaped input response.

In summary, the RNF approach is basically an open-loop scheme; the FFT block acts so slowly that its effect on the dynamics of the system is ignored. The purpose of RNF is to modify an arbitrary path so that the beam has little residual vibration. This scheme is easily appended to existing control schemes with a flexible manipulator of arbitrary design. 


\section{ADAPTIVE INTERFERENCE CANCELER}

The objective of the adaptive interference canceler (AIC) controller is to cancel the initial vibrations on the system by superimposing another vibrational wave. This idea is new to flexible-beam-type problems but has been used in other areas, such as noise reduction and electrocardiogram filtering problems. (See Widrow (1985) for specific details and for other application areas.) The error signal is physically the signal from the accelerometer and the output from the AIC controller is the torque drive signal to the actuator $\tau_{a}$ (see Fig. 5.)

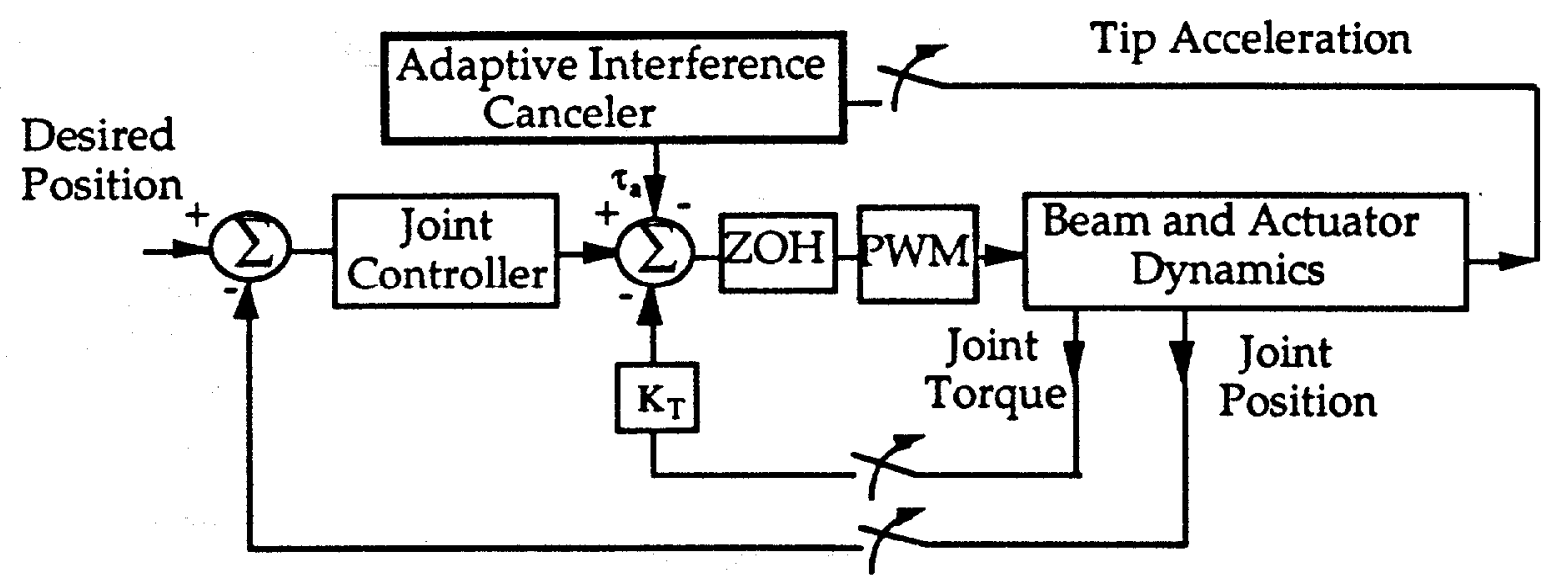

Fig. 5. Adaptive interference canceler block diagram.

Figure 6 is the error model for the AIC controller. Initial vibrations are assumed to be undamped sinusoids and are modeled as a cosine wave passing through an unknown transfer function block labeled $G_{\text {init }}$, which assigns the correct magnitude and phase shift to the cosine wave. The plant transfer function is also assumed to be unknown and is labeled as $G_{p}$.

To analyze the stability of the AIC, which is a nonlinear controller, it is assumed that the adaptation parameters $x_{\mathbf{c}}$ and $x_{\mathbf{s}}$ (see Fig. 6) vary slowly with respect to the vibrational wave. Furthermore, it is assumed that only the first modal frequency is of importance (higher modal frequencies are handled by adding additional software blocks, as shown in Fig. 6, in parallel at their associated modal frequencies) and that the first modal frequency is known exactly (i.e., $\omega_{0}=\omega_{1}$ ) by means of the FFT routine. From Fig. 6 , the error signal is

$$
\begin{aligned}
e=\operatorname{Re}\left\{\left[x_{s} G_{p}\left(j \omega_{0}\right) e^{-j \pi / 2}+x_{c} G_{p}\left(j \omega_{0}\right)\right] e^{j \omega_{0} t}\right\} \\
-\operatorname{Re}\left\{G_{\text {init }}\left(j \omega_{0}\right) e^{j \omega_{0} t}\right\} .
\end{aligned}
$$


The adaptation parameters are governed by

$$
\begin{aligned}
& \frac{d x_{s}}{d t}+\alpha x_{s}=K e \sin \omega_{b} t \\
& \frac{d x_{c}}{d t}+\alpha x_{c}=K e \cos \omega_{b} t
\end{aligned}
$$

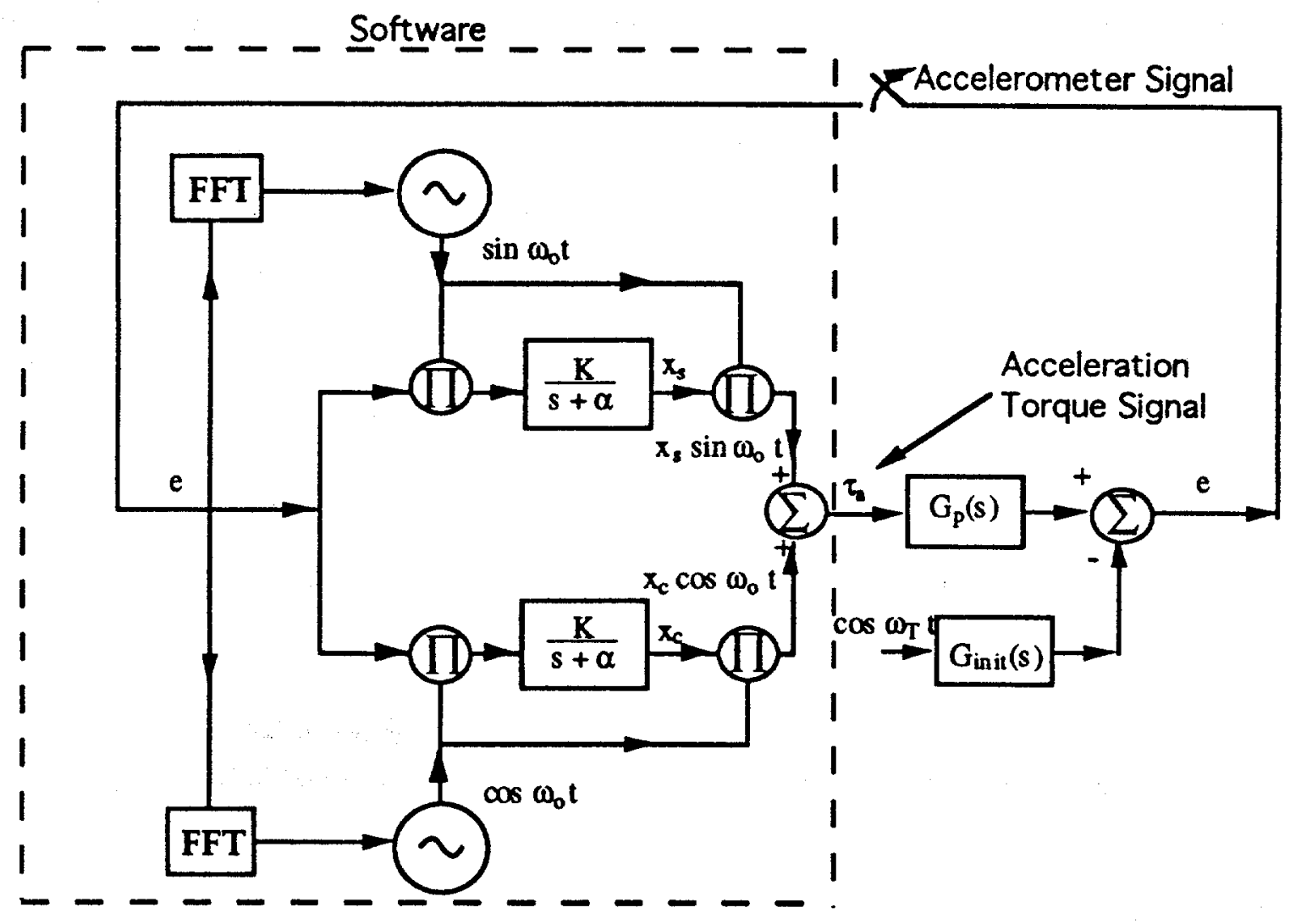

Fig. 6. Error model for the AIC controller.

Averaging techniques are used to analyze the stability of the controller. Because the adaptation parameters are assumed to vary slowly with respect to the vibrational waves, Eqs. (10a) and (10b) become

$$
\begin{aligned}
& \overline{x_{c}}+\alpha \overline{x_{c}}=K \operatorname{Avg}\left\{e \cos \omega_{b} t\right\} \\
& \overline{x_{s}}+\alpha \overline{x_{s}}=K \operatorname{Avg}\left\{e \sin \omega_{0} t\right\},
\end{aligned}
$$


where

$$
\begin{aligned}
& \operatorname{Avg}\{f(t)\} \equiv \frac{1}{T} \int_{0}^{T} f(t) d t \\
& T=\text { period of } \omega_{0} .
\end{aligned}
$$

Using the fact that

$$
\operatorname{Avg}\left(A \cos \left(\omega_{b} t+\varphi_{A}\right) B \cos \left(\omega_{0} t+\varphi_{B}\right)\right)=\frac{A B}{2} \cos \left(\varphi_{A}-\varphi_{B}\right),
$$

then

$$
\begin{gathered}
\operatorname{Avg}\left(e \cos \omega_{0} t\right\}=0.5 \overline{\bar{x}_{\delta}} \mid G_{p}\left(j \omega_{0}\right) \cos \left(-\pi / 2+\angle G_{p}\left(j \omega_{0}\right)\right) \\
+0.5 \overline{\bar{x}_{c}}\left|G_{p}\left(j \omega_{0}\right) \cos \left(\angle G_{p}\left(j \omega_{0}\right)\right)-0.5\right| G_{\text {init }}\left(j \omega_{0}\right) \cos \left(\angle G_{\text {init }}\left(j \omega_{0}\right)\right)
\end{gathered}
$$

and

$$
\begin{gathered}
\left.A v g\left\{e \sin \omega_{0}\right\}\right\}=0.5 \overline{x_{s}} \mid G_{p}\left(j \omega_{0}\right) \cos \left(-\pi+\angle G_{p}\left(j \omega_{b}\right)\right) \\
+0.5 \overline{x_{c}}\left|G_{p}\left(j \omega_{0}\right) \cos \left(\angle G_{p}\left(j \omega_{0}\right)-\pi / 2\right)-0.5\right| G_{\text {init }}\left(j \omega_{b}\right) \cos \left(\angle G_{\text {init }}\left(j \omega_{0}\right)-\pi / 2\right) .
\end{gathered}
$$

Using Eqs. (13a) and (13b), Eqs. (10a) and (10b) are rewritten as

$$
\begin{gathered}
\frac{d}{d t}\left[\overline{x_{s}}\right]=\left[\begin{array}{cc}
-\alpha-0.5 K / G_{p} \cos \left(\angle G_{p}\right) & 0.5 K / G_{p} \mid \sin \left(\angle G_{p}\right) \\
0.5 K / G_{p} \mid \sin \left(\angle G_{p}\right) & -\alpha+0.5 K\left|G_{p}\right| \cos \left(\angle G_{p}\right)
\end{array}\right]\left[\begin{array}{l}
\overline{x_{s}} \\
\overline{x_{c}}
\end{array}\right] \\
-0.5 K / G_{\text {init }}\left[\begin{array}{c}
\sin \left(\angle G_{\text {init }}\right) \\
\cos \left(\angle G_{\text {init }}\right)
\end{array}\right] .
\end{gathered}
$$

Equation (14) is a linear time-invariant differential equation. The eigenvalues of this set of equations can be shown to be

$$
\lambda=-\alpha \pm 0.5 \mathrm{~K} \mid \mathrm{G}_{\mathrm{p}}\left(j \omega_{\mathrm{o}} \mid\right) .
$$

For a stable system, the eigenvalues must lie in the left half plane. Even though the system plant $G_{p}$ is assumed to be unknown, stability will be achieved if $\alpha>0.5 \mathrm{~K} \mid \mathrm{G}_{\mathrm{p}}\left(j \omega_{\mathrm{o}}\right)$ for $\mathrm{K}>0$ and $\alpha>0$. Furthermore, while $\omega_{0}$ is the first modal frequency of the plant $G_{p}$, there will be some small structural damping that will prevent $\mid G_{p}\left(j \omega_{0} \mid\right.$ from becoming unstable. The equilibrium point for Eq. (4), when $\alpha=0$, is found by setting the left side of Eq. (14) to zero. This equilibrium point can be shown to force $e(t)$ in Eq. (9) to zero. Unfortunately, $\alpha$ must be greater than zero for stability to be satisfied. For nonzero $\alpha$, the equilibrium point forces 
e(t) to a smaller, but nonzero value, during each cycle period. Experimental results shows that eventually $e(t)$ goes to zero.

The flexible manipulator is again slewed $20^{\circ}$ for a duration of $0.25 \mathrm{~s}$. Tip feedback is obtained from an accelerometer at the distal end of the beam. The shaping filter is turned off. Figure 7 shows the plot of the adaptive-feedback response compared with the ramped response. A significant reduction is clearly shown.

In summary, a simple AIC controller was introduced. The advantage of accelerometer feedback from the tip of the beam is the simplicity of modifying any existing design. Clearly, beam damping was increased.

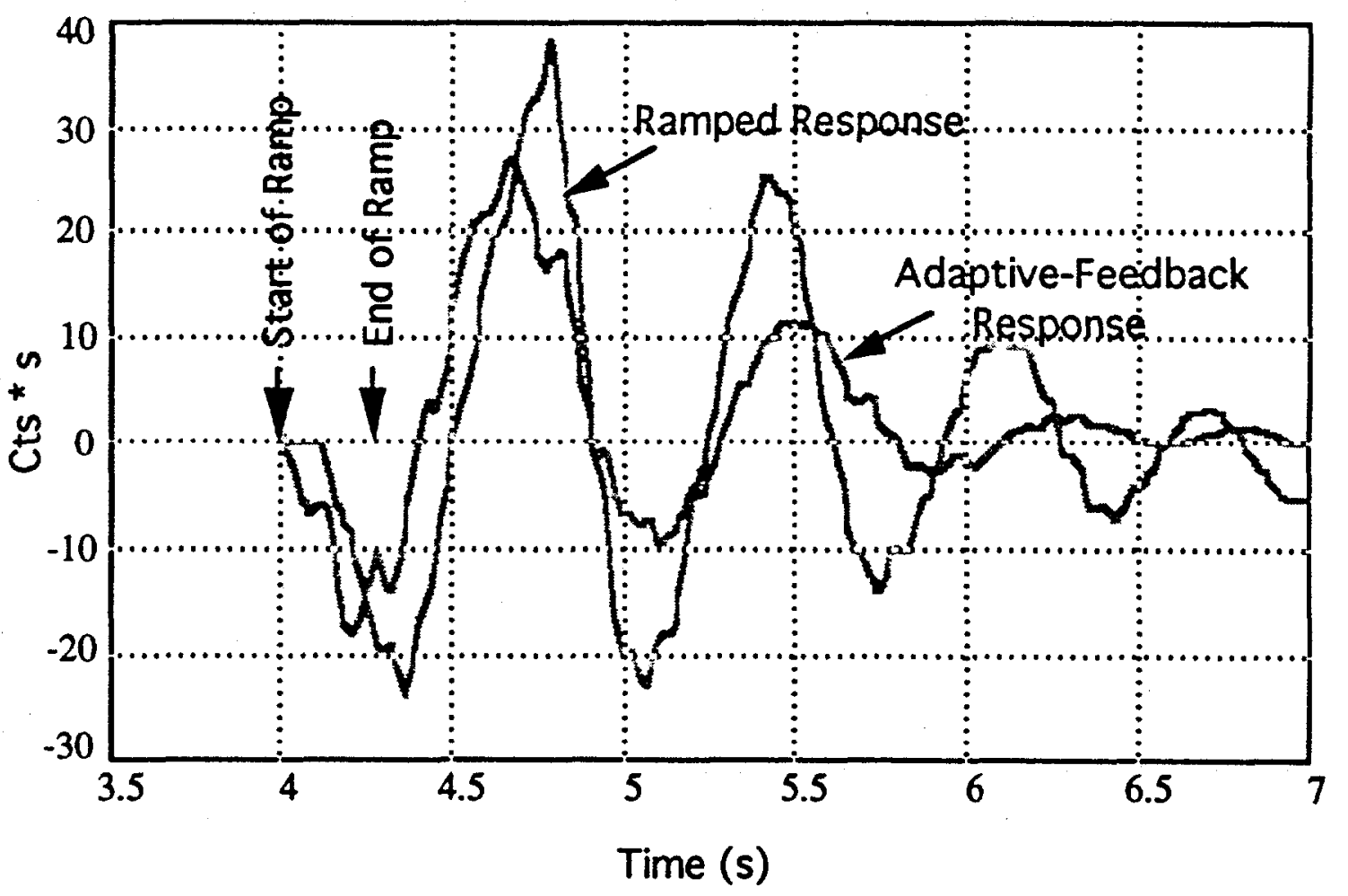

Fig. 7. Adaptive-feedback response of the AIC controller. 


\section{ACCELERATION FEEDBACK}

For the last controller, the acceleration sensor is again used, but classical linear compensation is implemented. The block diagram of this controller is shown in Fig. 8.

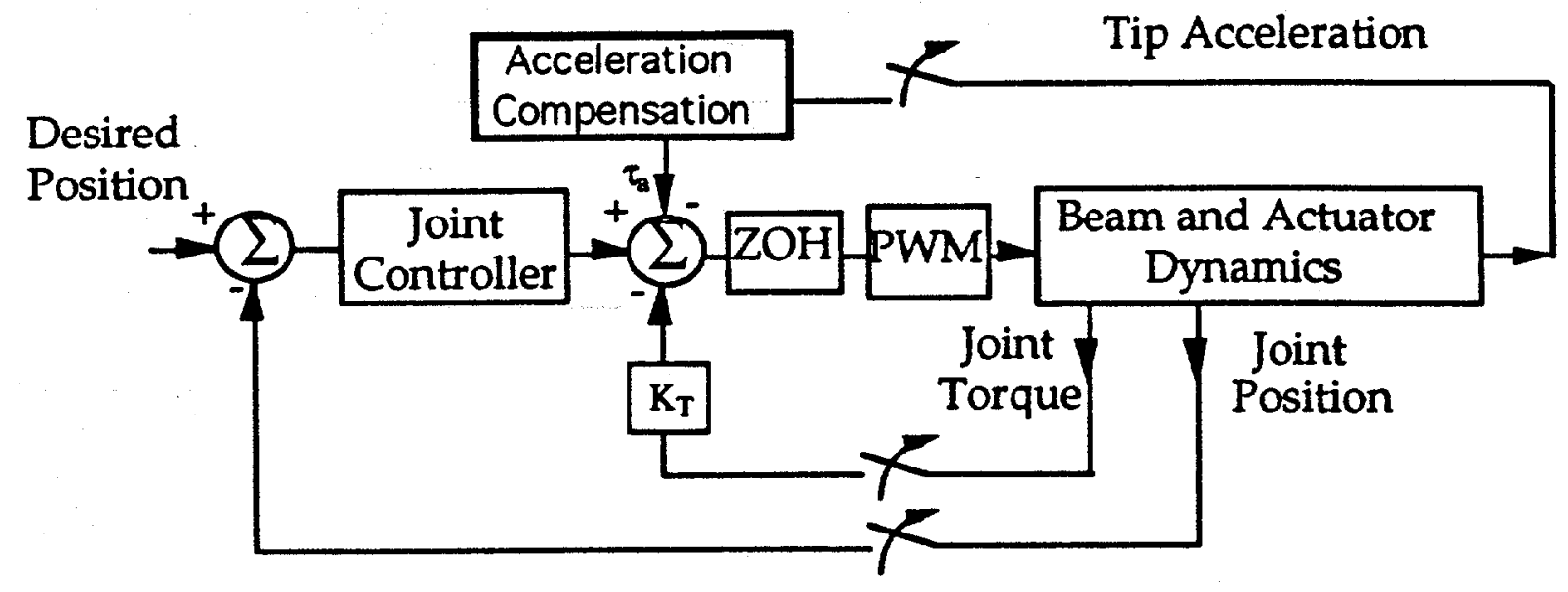

Fig. 8. Acceleration feedback block diagram.

The flexible manipulator was slewed $20^{\circ}$ for a duration of $0.25 \mathrm{~s}$. An acceleration feedback scheme was implemented along with local joint and torque feedback. Tip feedback is employed by an accelerometer at the distal end of the beam. The shaping filter is turned off. A non-minimum phase compensator is applied to the tip acceleration signal and is fed back at the actuator. This scheme was carefully tuned to avoid potential instabilities due to load variations. The dynamic model for the flexible manipulator and the joint and acceleration controllers are shown in Fig. 9. A local torque loop is also running but is not shown in the diagram because its function is to diminish the effect of the nonlinear dynamics of the system. 


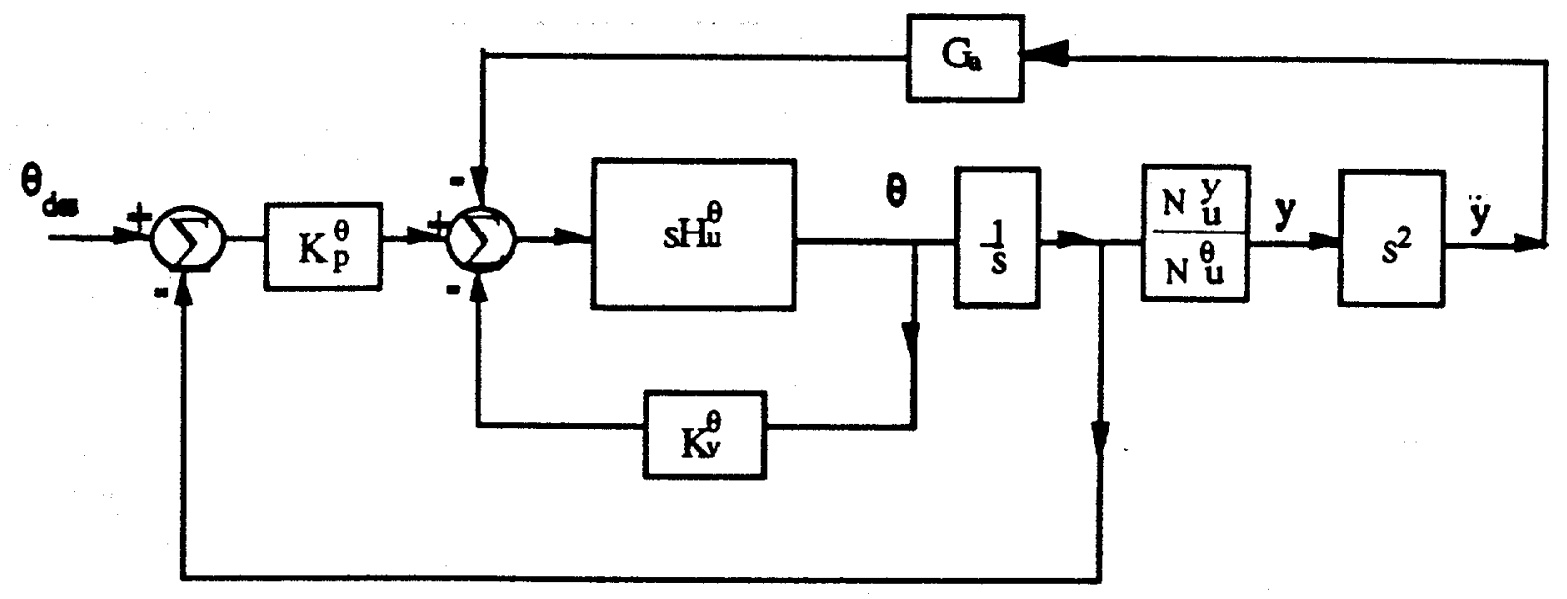

Fig. 9. Basic block diagram of flexible manipulator dynamics and acceleration controller.

For stability analysis, the above block diagram can be cast as the following root-locus problem:

$$
\frac{G_{1} N_{u}^{y} s^{2} G_{a}}{N_{u}^{\theta}\left(s+K_{p} G_{1}\right)}=-1
$$

where

$$
\mathrm{G}_{1}=\frac{s \mathrm{H}_{\mathrm{u}}^{\theta}}{1+s \mathrm{~K}_{\mathrm{v}}^{\theta} \mathrm{H}_{\mathrm{u}}^{\theta}}
$$

The accelerometer compensator was designed as an all-pass filter (i.e., $G_{a}=K_{\text {all }}\left(\frac{s}{z_{\text {all }}}+1\right) /\left(\frac{s}{p_{\text {all }}}+1\right)$ and $\left.z_{\text {all }}=-p_{\text {all }}\right)$ with the associated root-locus plot shown in Fig. 10.

The first three modal frequencies are included in this root-locus plot; however, only the lower frequency poles are shown because these are the dominant ones. Further, the scales are changed in these plots because the purpose of these plots is only to convey the general movement of the system poles. 


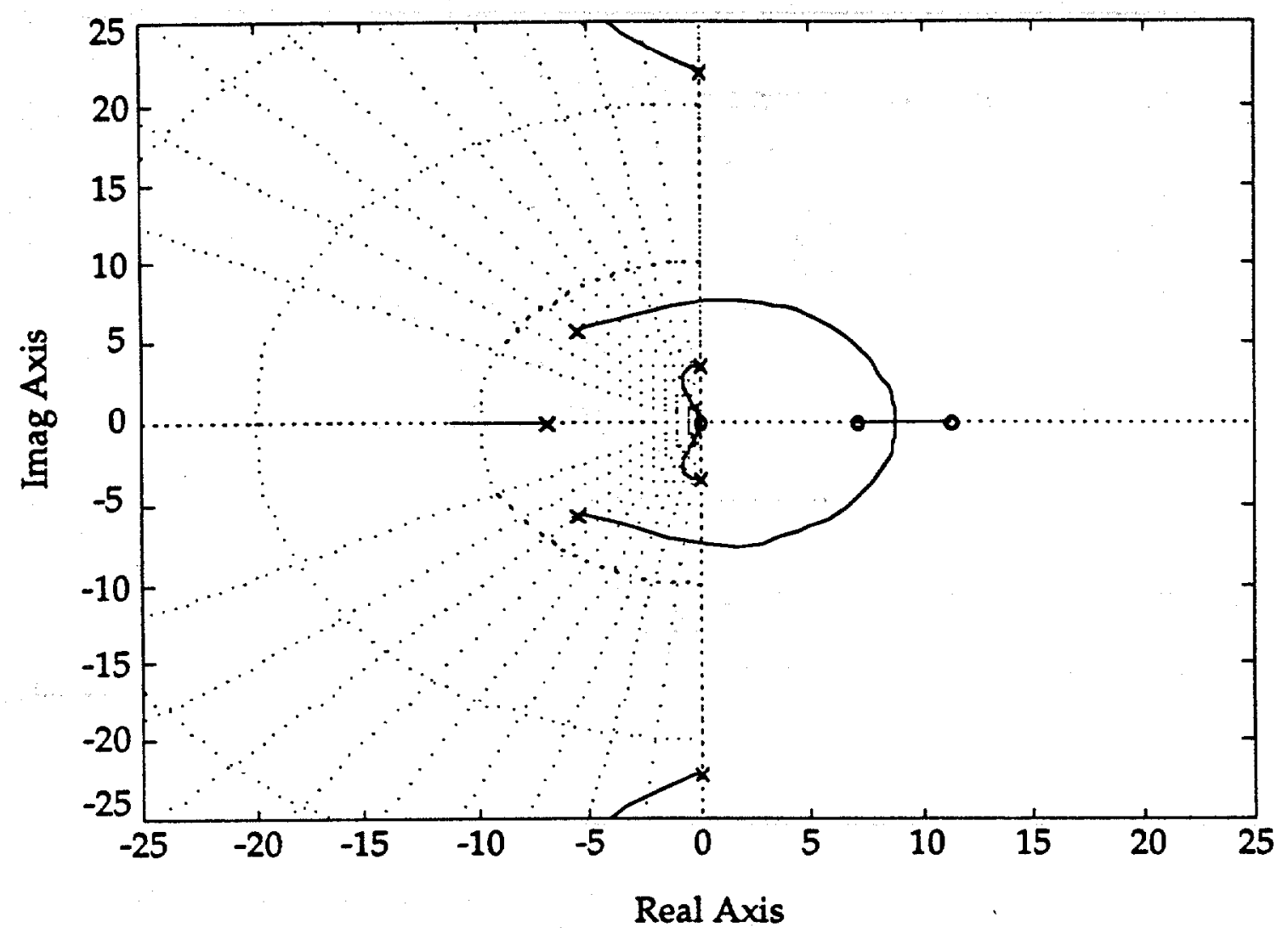

Fig. 10. Root-locus plot of the block diagram in Fig. 9.

As the gain of the accelerometer compensator $\mathrm{K}_{\mathrm{all}}$ is varied, the system poles move as shown in Fig. 10. Stability is maintained by limiting $\mathbf{K}_{\text {all }}$ so that all the poles stay in the left half plane. In summary, the time response and its comparison to the ramped response are shown in Fig. 11. Clearly, the accelerometer response damps out residual vibrations. 


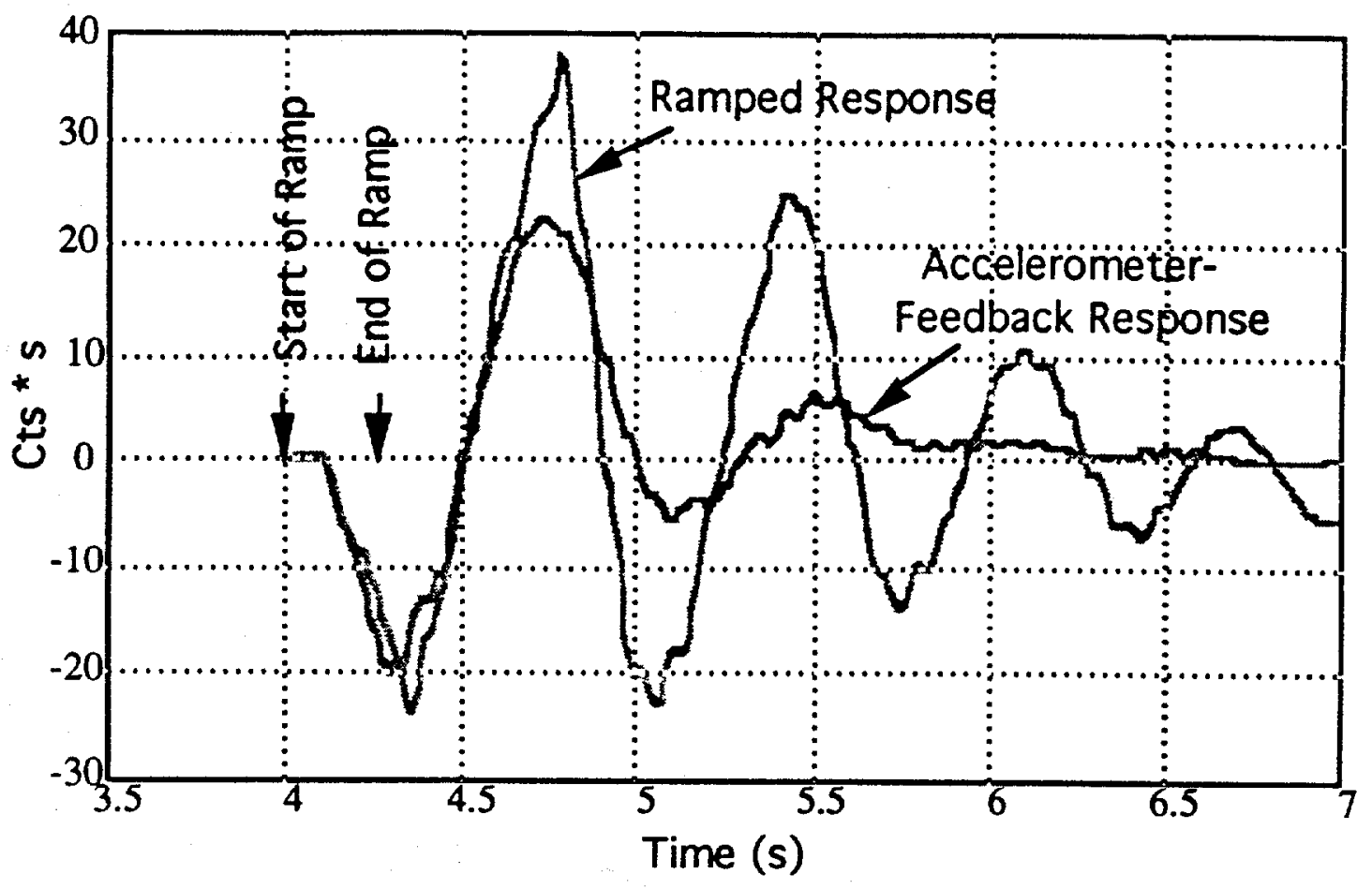

Fig. 11. Time response of the acceleration controller. 


\section{CONCLUSION AND SUMMARY}

Three controllers were implemented on a single flexible manipulator. All three controllers have a joint proportional-derivative controller and a base torque feedback loop. The first controller, an open loop scheme using RNFs, can be used by itself or with the other two controllers. Its function is to modify the command signal to reduce the residual vibration modes at the flexible manipulator tip. Input commands can be modified in a real-time environment by means of conventional microprocessors. Modal frequencies are determined by means of an accelerometer sensor. Accelerometers are fairly easy to instrument on any mechanical structure, adding to their advantage over other sensors, such as strain gauges. Because of measurement noise, the RNFs are designed to be robust with regard to the system's natural frequencies and damping ratios. The system can be easily extended to multiple-link manipulators. The second controller was an adaptive controller that is based on interference cancellation methods. The basic idea is that vibrational waves are generated by the actuator to cancel any tip vibrations. Tip vibrations are measured by means of an accelerometer mounted on the distal end of the beam. The third controller used a linear compensator in conjunction with an accelerometer feedback scheme. All three controllers were successfully implemented and performed well on a single flexible manipulator. 


\section{REFERENCES}

1. Meirovitch, L. 1967. Analytical Methods in Vibrations, Macmillan, New York.

2. Bhat, S. P., and Miu, D. K. December 1990. "Precise Point-to-Point Positioning Control of Flexible Structures," L. of Dynamic Systems. Measurement and Control, $112(4), 667-674$.

3. Jayasuriya, S., and Choura, S. 1990. "Active Quenching of a Set of Predetermined Vibratory Modes of a Beam by a Single Fixed Point Actuator," Int. ]. Control, $51(2), 445-67$.

4. Jansen, J. F., et al. 1992. Long-Reach Manipulation for Waste Storage Tank Remediation FY 1992 Report. ORNL/TM-11999, Martin Marietta Energy Systems, Inc., Oak Ridge National Laboratory.

5. Singhose, W. E., Seering, W. P., and Singer, N. C. 1990. "Shaping Inputs to Reduce Vibration: A Vector Diagram Approach," Proceedings of the 1990 IEEE Conference on Robotics and Automation, Cincinnati, Ohio, 922-927, May 1990.

6. Murphy, B. R., and Watanabe, I. April 1992. "Digital Shaping Filters for Reducing Machine Vibration," IEEE Trans. Robotics and Automation, 8 (2), 285-89.

7. Widrow, B., and Stearns, S. D. 1985. Adaptive Signal Processing Prentice-Hall, Englewood Cliffs, N. J. 
ORNL/TM-12198

\section{INTERNAL DISTRIBUTION}

1-10. S. M. Babcock

11. J. B. Berry

12-21. B. L. Burks

22. M. J. Haire

23. D. C. Haley

24-25. W. R. Hamel

26. J. N. Herndon

27-58. J. F. Jansen

59-63. R. L. Kress

64. C. T. Kring

65. D.S. Kwon

66. A. P. Malinauskas

67. R. C. Mann

68. S. March-Leuba
69-71. S. A. Meacham

72. M. W. Noakes

73. F. G. Pin

74. K. E. Plummer

75. B. S. Richardson

76. S. L. Schrock

77. J. O. Stiegler

78. F. J. Sweeney

79. H. R. Yook

80. ORNL Central Research Library

81. ORNL Document Reference Section 82-83. ORNL Laboratory Records

84. ORNL Laboratory Records RC

85. ORNL Patent Section

\section{EXTERNAL DISTRIBUTION}

86. Clinton Bastin, Manager, LMR Reprocessing Projects, Division of Fuels and Reprocessing, Office of Facilities, Fuel Cycle, and Test Programs, NE-471, Department of Energy, Washington, DC 20545

87. D. W. Bennett, Pacific Northwest Laboratories, P.O. Box 999, Richland, WA 99352

88. L. F. Blankner, Fusion and Nuclear Technology Branch, Energy Programs Division, Department of Energy, P.O. Box 2008, Oak Ridge, TN 37831-6269

89. S. A. Couture, Lawrence Livermore National Laboratory, P.O. Box 808, L-437, Livermore, CA 64551

90. M. S. Evans, Pacific Northwest Laboratories, P.O. Box 999, Richland, WA 99352

91. R. M. Hollen, Los Alamos National Laboratory, P.O. Box 1663, MS J-580, Los Alamos, NM 87545 
92. R. W. Harrigan, Sandia National Laboratories, P.O. Box 5800, Division 1414, Albuquerque, NM 87185

93. D. L. Jacoboski, Fernald Management Company of Ohio, P.O. Box 398704, Cincinnati, OH 45239-8704

94. W. Jaquish, Westinghouse Hanford Company, P.O. Box 1970, LO-18, Richland, WA 99352

95. L. Kostelnik, Westinghouse Idaho Nuclear Company, P.O. Box 4000, Idaho Falls, ID 83403-5104

96. C. R. Ward, Westinghouse Savannah River Company, Building 773-A, D-1145, Aiken, SC 29808

97. A. P. Williams, Westinghouse Hanford Company, P.O. Box 1970, LO-18, Richland, WA 99352

98. L. W. Yarbrough, Department of Energy, 12800 Middlebrook Road, MS EM-55, Trevion II, Washington, DC 20874

99. Office of Assistant Manager for Energy Research and Development, DOE Oak Ridge Field Office, P.O. Box 2008, Oak Ridge, TN 37831-6269

100-101. Office of Scientific and Technical Information, DOE Oak Ridge Field Office, P.O. Box 62, Oak Ridge, TN 37831 